\title{
Motion estimation in PET-MRI based on dual registration: preliminary results for human data
}

Michael Fieseler ${ }^{1,2^{*}}$, Thomas Kösters ${ }^{3}$, Christopher Glielmi ${ }^{4}$, Fernando Boada ${ }^{3}$, David Faul ${ }^{4}$, Matthias Fenchel ${ }^{5}$, Robert Grimm ${ }^{6}$, Xiaoyi Jiang ${ }^{1,2}$, Klaus P Schäfers $^{1}$

From PSMR14: 3rd Conference in PET/MR and SPECT/MR

Kos Island, Greece. 19-21 May 2014

${ }^{1}$ European Institute for Molecular Imaging, University of Münster, Münster, Germany

In current motion correction approaches in PET-MRI, motion information from PET data is neglected. We present an approach where PET and MRI data are used for motion estimation simultaneously. The presented approach has been evaluated on phantom data before [1]. Here, we present first results for human PET-MRI data.

The registration functional for dual registration is given by

$$
\mathrm{J}(\mathrm{y})=\mathrm{D}\left(\mathrm{T}_{\mathrm{MR}}(\mathrm{y}), \mathrm{R}_{\mathrm{MR}}\right)+\beta-\mathrm{D}\left(\mathrm{T}_{\mathrm{PET}}(\mathrm{y}), \mathrm{R}_{\mathrm{PET}}\right)+\alpha-\mathrm{S}(\mathrm{y})
$$

Here, $R_{M R}$ and $R_{P E T}$ denote two reference volumes and $T_{M R}$ and $T_{\text {PET }}$ the template volumes to be registered, $\mathrm{D}$ is a distance functional, and $\mathrm{S}$ is a regularizer. The scalar value $\beta$ allows to weight the influence of the data term for PET [1]. The functional has been implemented using the FAIR toolbox [3].

Five patients were scanned following a clinical FDG scan. A self-gated radial VIBE sequence [2] and PET Listmode data were acquired. The datasets were re-binned into 5 coinciding PET and MRI phases (gates).

Registration were computed for $\beta \in\{0,0.5,1,2\}$, $\alpha$ was chosen empirically as $\alpha=20$.

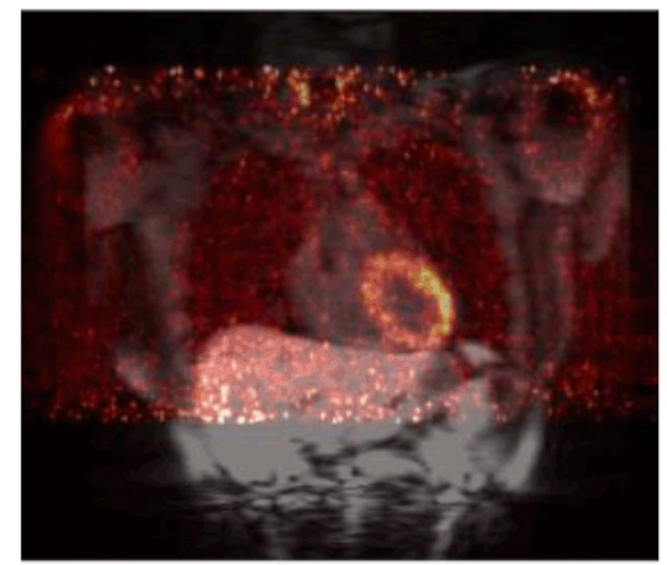

(a) Dataset 4, coronal overlay

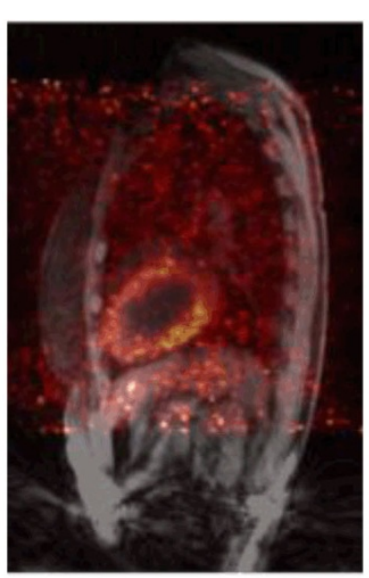

(b) Dataset 4, sagital overlay

Figure 1 Overlay of PET and MR data for dataset 4, first respiratory phase (gate).

c) 2014 Fieseler et al; licensee Springer This is an Open Access article distributed under the terms of the Creative Commons Attribution License (http://creativecommons.org/licenses/by/4.0), which permits unrestricted use, distribution, and reproduction in any medium, provided the original work is properly cited. 

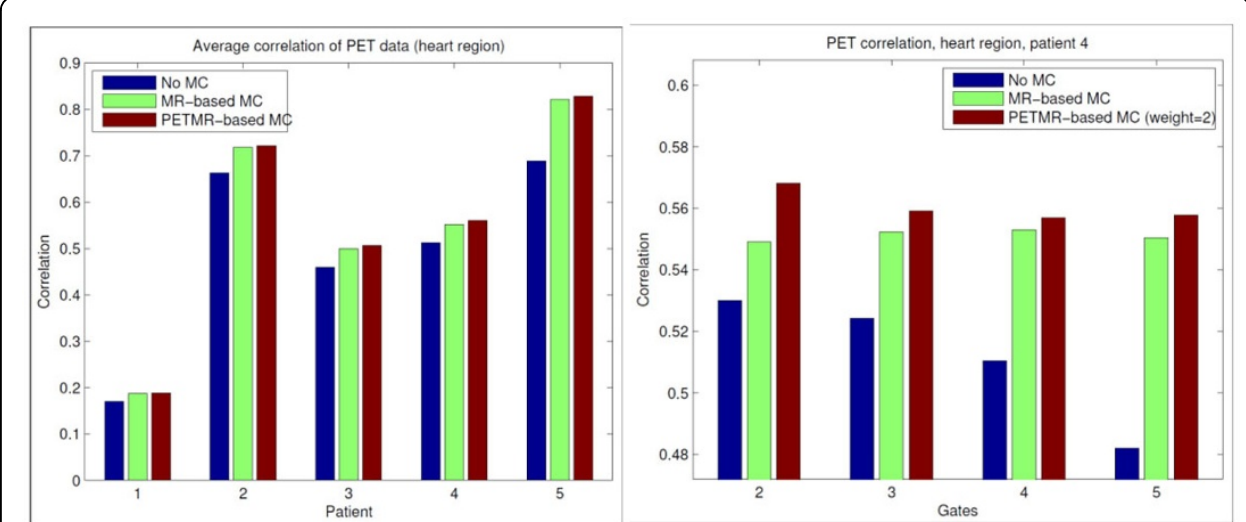

a) Dataset 4 , correlation per gate

b) Average correlation for all 5 datasets

Figure 2 Correlation values for PET data. (a) Correlation values for the heart region in gates 2 to 5 for dataset number 4. (b) Average correlation values for all patients

Correlation coefficients were computed for the heart region.

In Figure 2a we show correlation values for each gate of dataset 4. In all gates the correlation of the PET data is improved using the joint motion estimation approach using a weight of $\beta=2$. In $2 \mathrm{~b}$ average correlation values of all gates are shown for all datasets processed.

We have shown that using a joint motion estimation approach the correlation of PET data is improved compared to an estimation of the motion solely on MRI data. Currently, we are evaluating motion-correcting reconstructions using the motion estimates from the proposed method.

Authors' details

${ }^{1}$ European Institute for Molecular Imaging, University of Münster, Münster, Germany. ${ }^{2}$ Department of Computer Science, University of Münster, Münster, Germany. ${ }^{3}$ Center for Advanced Imaging Innovation and Research, NYU Langone Medical Center, New York, USA. ${ }^{4}$ Siemens Medical Solutions USA, New York, USA. ${ }^{5}$ Siemens AG, Healthcare Sector, Germany. ${ }^{6}$ Pattern Recognition Lab, University of Erlangen-Nürnberg, Erlangen, Germany.

Published: 29 July 2014

\section{References}

1. Fieseler M, Gigengack F, Jiang X, Schäfers KP: Motion correction of whole-body PET data with a joint PET-MRI registration functional. BioMedical Engineering OnLine 2014, 13(Suppl 1):S2.

2. Grimm R, Fürst S, Dregely I, Forman C, Hutter JM, Ziegler SI, Nekolla S, Kiefer B, Schwaiger M, Hornegger J, et al: Selfgated radial MRI for respiratory motion compensation on hybrid PET/MR systems. Medical Image Computing and Computer-Assisted Intervention- MICCAI 2013, 17-24

3. Modersitzki J: Fair: Flexible Algorithms for Image Registration (Fundamentals of Algorithms). Society for Industrial and Applied Mathematics Philadelphia; 2009.

doi:10.1186/2197-7364-1-S1-A39

Cite this article as: Fieseler et al:: Motion estimation in PET-MRI based on dual registration: preliminary results for human data. EJNMMI Physics 2014 1(Suppl 1):A39. 